\title{
Renormalization-Group Improved Calculation of Top-Quark Production Near Threshold
}

\author{
A. H. Hoang, ${ }^{1}$ A. V. Manohar, ${ }^{2}$ I. W. Stewart, ${ }^{2}$ and T. Teubner ${ }^{3}$ \\ ${ }^{1}$ Theory Division, CERN, CH-1211 Geneva 23, Switzerland \\ ${ }^{2}$ Department of Physics, UCSD, 9500 Gilman Drive, La Jolla, California 92093-0319 \\ ${ }^{2}$ Institut für Theoretische Physik E, RWTH Aachen, D-52056 Aachen, Germany
}

(Received 21 November 2000)

\begin{abstract}
The top-quark cross section close to threshold in $e^{+} e^{-}$annihilation is computed including the summation of logarithms of the velocity at next-to-next-to-leading-logarithmic order in QCD. The remaining theoretical uncertainty in the normalization of the total cross section is at the few-percent level, an order of magnitude smaller than in previous next-to-next-to-leading order calculations. This uncertainty is smaller than the effects of a light standard-model Higgs boson.
\end{abstract}

DOI: 10.1103/PhysRevLett.86.1951

Top-quark pair production close to threshold is a major part of the top-quark physics program at a future lepton collider. Near threshold the top-quark velocity $v$ is small and the presence of Coulomb singularities make the summation of terms proportional to $\alpha_{s} / v$ mandatory. The top-quark width, $\Gamma_{t} \approx 1.5 \mathrm{GeV} \gg \Lambda_{\mathrm{QCD}}$, serves as an infrared cutoff, allowing for the use of perturbative methods to calculate the nonrelativistic top-antitop dynamics to a high degree of precision.

Recent next-to-next-to-leading-order (NNLO) QCD calculations of the total cross section $\sigma_{t \bar{t}}$ showed that the top-quark mass can be determined with uncertainties below $200 \mathrm{MeV}$, but indicated that the strong coupling, the top Yukawa coupling, and $\Gamma_{t}$ cannot be measured with good precision due to large theoretical normalization uncertainties of about $20 \%$ [1]. A common feature of all recent NNLO QCD calculations is that they are fixed order calculations, i.e., the running from the hard scale $m_{t}$ down to the nonrelativistic scales which govern the dynamics of the top-antitop system was not taken into account. For $t \bar{t}$, $v \sim 0.15$ so that logarithms of ratios of $m_{t} \sim 175 \mathrm{GeV}$, $m_{t} v \sim 25 \mathrm{GeV}$, and $m_{t} v^{2} \sim 4 \mathrm{GeV}$ are not small, and the renormalization-group evolution is significant.

In this Letter we calculate the photon-induced $t \bar{t}$ production cross section in the framework of vNRQCD using the velocity renormalization group (VRG) [2]. The calculation includes a summation of logarithms of the ratios of the scales $m_{t}, m_{t} v$, and $m_{t} v^{2}$ at next-to-next-to-leading order. The VRG-improved computation has a scale uncertainty of $2 \%-3 \%$ at the peak of the cross section (without initial state radiation and beam smearing effects). An improvement in the convergence of the expansion is also found. Measurements of the strong coupling, the top Yukawa coupling, and the top-quark width appear feasible with small theoretical uncertainties.

The expansion for the normalized cross section $R=$ $\sigma_{\bar{t} \bar{t}} / \sigma_{\mu^{+} \mu^{-}}$takes the form
PACS numbers: 14.65.Ha, 12.38.Cy

$$
R=v \sum_{k, i}\left(\frac{\alpha_{s}}{v}\right)^{k}\left(\alpha_{s} \ln v\right)^{i} \begin{cases}1 & (\mathrm{LL}), \\ \alpha_{s}, v & \text { (NLL), } \\ \alpha_{s}^{2}, \alpha_{s} v, v^{2} & \text { (NNLL) }\end{cases}
$$

The free quark cross section is of order $v$. The Coulomb summation of powers of $\alpha_{s} / v$ and the VRG summation of powers of $\alpha_{s} \ln v$ are the sums over $k$ and $i$, respectively. Terms in the cross section at leading-logarithmic (LL), next-to-leading-logarithmic (NLL), and next-tonext-to-leading-logarithmic (NNLL) order are indicated in Eq. (1).

vNRQCD [2] is an effective field theory which describes nonrelativistic heavy quarks with mass $m$ interacting with soft gluons with four-momenta $k^{\mu} \sim m v$ and ultrasoft gluons with $k^{\mu} \sim m v^{2}$, where $m v^{2}$ is larger than $\Lambda_{\mathrm{QCD}}$. For loop integrations over soft energies and momenta the modified minimal subtraction ( $\overline{\mathrm{MS}}$ ) scale is $\mu_{S}=m \nu$, whereas for loop integrations with ultrasoft energies and momenta it is $\mu_{U}=m \nu^{2}$. The subtraction velocity $\nu$ correlates $\mu_{S}$ and $\mu_{U}$ and is used instead of the $\overline{\mathrm{MS}}$ scale parameter $\mu$. The correlation is mandatory since energy and momentum are related through the quark equations of motion. In QED this has been shown to be necessary to reproduce through running the $(\ln \alpha)^{k}, k \geq 2$ contributions in Lamb shifts, hyperfine splittings, and corrections to the ortho and para positronium decay widths [3].

Lowering $\nu$ from $\nu=1$ to the quark velocity $v$ sums all large logarithms involving the soft and ultrasoft scales into the Wilson coefficients of the vNRQCD operators. At NNLL this includes logarithms originating from radiation effects. Once $\nu$ is lowered to $v$, power counting shows that matrix elements with ultrasoft gluons do not have to be taken into account at NNLL for the description of a heavy quark pair in a color singlet state. Thus, after lowering $\nu$ to order $\alpha_{s}$ (since $v \sim \alpha_{s}$ in a Coulombic system) the NNLL equation of motion of a color singlet quark-antiquark system is a conventional two-body Schrödinger equation.

In momentum space the NNLL Schrödinger equation reads

$\left[\frac{\boldsymbol{p}^{2}}{m}-\frac{\boldsymbol{p}^{4}}{4 m^{3}}-E\right] \tilde{G}\left(\boldsymbol{p}, \boldsymbol{p}^{\prime}\right)+\int D^{n} \boldsymbol{q} \mu_{S}^{2 \epsilon} \tilde{V}(\boldsymbol{p}, \boldsymbol{q}) \tilde{G}\left(\boldsymbol{q}, \boldsymbol{p}^{\prime}\right)=(2 \pi)^{n} \delta^{(n)}\left(\boldsymbol{p}-\boldsymbol{p}^{\prime}\right)$, 
where $m$ is the heavy quark pole mass, $E \equiv(\sqrt{s}-2 m)$, $n \equiv 3-2 \epsilon$, and $D^{n} \boldsymbol{q} \equiv e^{\epsilon \gamma_{E}}(4 \pi)^{-\epsilon} d^{n} \boldsymbol{q} /(2 \pi)^{n}$. The potential for the quark-antiquark pair in a ${ }^{3} S_{1}$ state (relevant for top production through a virtual photon) is

$$
\tilde{V}(\boldsymbol{p}, \boldsymbol{q})=\tilde{V}_{c}(\boldsymbol{p}, \boldsymbol{q})+\tilde{V}_{k}(\boldsymbol{p}, \boldsymbol{q})+\tilde{V}_{\delta}(\boldsymbol{p}, \boldsymbol{q})+\tilde{V}_{r}(\boldsymbol{p}, \boldsymbol{q}),
$$

where $\left[a_{s}=\alpha_{s}\left(\mu_{S}\right), L=\ln \left(\boldsymbol{k}^{2} / \mu_{S}^{2}\right), \boldsymbol{k}=\boldsymbol{p}-\boldsymbol{q}\right]$

$$
\begin{aligned}
\tilde{V}_{c}(\boldsymbol{p}, \boldsymbol{q})= & \frac{\mathcal{V}_{c}(\nu)}{\boldsymbol{k}^{2}}-\frac{4 \pi C_{F} a_{s}}{\boldsymbol{k}^{2}} \\
& \times\left\{\frac{a_{s}}{4 \pi}\left[-\beta_{0} L+a_{1}\right]+\left(\frac{a_{s}}{4 \pi}\right)^{2}\right. \\
& \left.\times\left[\beta_{0}^{2} L^{2}-\left(2 \beta_{0} a_{1}+\beta_{1}\right) L+a_{2}\right]\right\}, \\
\tilde{V}_{k}(\boldsymbol{p}, \boldsymbol{q})= & \frac{\pi^{2}}{m|\boldsymbol{k}|} \mathcal{V}_{k}(\nu), \\
\tilde{V}_{\delta}(\boldsymbol{p}, \boldsymbol{q})= & \frac{\mathcal{V}_{2}(\nu)+2 \mathcal{V}_{s}(\nu)}{m^{2}}, \\
\tilde{V}_{r}(\boldsymbol{p}, \boldsymbol{q})= & \frac{\left(\boldsymbol{p}^{2}+\boldsymbol{q}^{2}\right)}{2 m^{2} \boldsymbol{k}^{2}} \mathcal{V}_{r}(\nu) .
\end{aligned}
$$

The potentials arise from the four-quark matrix elements of potential-type operators and from time-ordered products of operators describing interactions with soft gluons. The coefficients of the potentials at the hard scale, $\mathcal{V}_{i}(\nu=1)$, are obtained with on-shell matching. For $\nu \sim v$ the coefficients contain the summation of all $\ln v$ terms. The velocity counting of each potential in Eq. (2) is equivalent to the counting in the Schrödinger equation in previous NNLO calculations (see, e.g., Ref. [4]). The coefficient $\mathcal{V}_{c}(\nu)$ in the Coulomb potential, $\tilde{V}_{c}$, was determined in Ref. [5] at NNLL order. The second term in Eq. (4) con-

tains the one- and two-loop corrections to the Coulomb potential [6]. In vNRQCD it arises from the time-ordered product of the lowest order operators describing the interaction of quarks with soft gluons. The couplings in this time-ordered product are $\alpha_{s}\left(\mu_{S}\right)$ and evolve with the QCD $\beta$ function. The potential $\tilde{V}_{k}$ leads to terms in the cross section that are $v^{2}$ suppressed. The coefficient $\mathcal{V}_{k}$ has a matching value of order $\alpha_{s}^{2}$ [7], so its NLL evolution from Ref. [8] is needed. The potentials $\tilde{V}_{\delta}$ and $\tilde{V}_{r}$ also lead to terms in the cross section that are $v^{2}$ suppressed and have order $\alpha_{s}$ coefficients $\mathcal{V}_{2, s, r}$ generated at tree level. Their evolution is needed only at LL order and can be found in Ref. [9].

To describe vector current induced quark-antiquark production close to threshold at NNLL order we also need the Wilson coefficients of the dimension-three ${ }^{3} S_{1}$ currents at NNLL order and the corresponding dimension-five currents at LL order. The nonrelativistic current for production is $\boldsymbol{J}_{\boldsymbol{p}}=c_{1} \boldsymbol{O}_{\boldsymbol{p}, 1}+c_{2} \boldsymbol{O}_{\boldsymbol{p}, 2}$, where

$$
\begin{aligned}
& \boldsymbol{O}_{p, 1}=\psi_{p}^{\dagger} \boldsymbol{\sigma}\left(i \sigma_{2}\right) \chi_{-p}^{*}, \\
& \boldsymbol{O}_{p, 2}=\frac{1}{m^{2}} \psi_{p}^{\dagger}\left(\boldsymbol{p}^{2} \boldsymbol{\sigma}\right)\left(i \sigma_{2}\right) \chi_{-\boldsymbol{p}}^{*} .
\end{aligned}
$$

Spin and color indices are suppressed. There is another dimension-five current describing $D$-wave production which does not contribute at this order. The corresponding annihilation currents $\boldsymbol{O}_{\boldsymbol{p}, 1,2}^{\dagger}$ are obtained by complex conjugation. The matching condition for the Wilson coefficient $c_{1}$ at the hard scale needs to be known at order $\alpha_{s}^{2}$, and the Wilson coefficient $c_{2}$ needs to be known at the Born level. The value of $c_{1}(\nu=1)$ can be determined from matching the two-loop result for the quark-antiquark production amplitude close to threshold in full QCD [10] to the corresponding amplitude in vNRQCD. We find

$$
\begin{aligned}
c_{1}(1)= & 1-2 C_{F} \frac{\alpha_{s}(m)}{\pi}+\alpha_{s}^{2}(m)\left[C_{F}^{2}\left(\frac{\ln 2}{12}-\frac{25}{24}-\frac{2}{\pi^{2}}\right)+C_{A} C_{F}(\ln 2-1)+\frac{\kappa}{2}\right], \\
\kappa= & C_{F}^{2}\left[\frac{1}{\pi^{2}}\left(\frac{39}{4}-\zeta_{3}\right)+\frac{4}{3} \ln 2-\frac{35}{18}\right]-C_{A} C_{F}\left[\frac{1}{\pi^{2}}\left(\frac{151}{36}+\frac{13}{2} \zeta_{3}\right)+\frac{8}{3} \ln 2-\frac{179}{72}\right] \\
& +C_{F} T_{F}\left[\frac{4}{9}\left(\frac{11}{\pi^{2}}-1\right)\right]+C_{F} T_{F} n_{l}\left[\frac{11}{9 \pi^{2}}\right] .
\end{aligned}
$$

The two-loop result for $c_{1}(1)$ is scheme dependent and our result differs from the hard contribution obtained from the threshold expansion [10,11].

The LL anomalous dimension for $c_{1}$ is zero. The evolution of $c_{1}$ for $\nu<1$ at NLL order has been determined analytically in Ref. [8] by solving [2]

$$
\frac{\nu}{c_{1}} \frac{\partial}{\partial \nu} c_{1}=-\frac{\mathcal{V}_{c}}{16 \pi^{2}}\left(\frac{\mathcal{V}_{c}}{4}+\mathcal{V}_{2}+\mathcal{V}_{r}+2 \mathcal{V}_{s}\right)+\frac{\mathcal{V}_{k}}{2}
$$

The summed logarithms in $c_{1}\left(\nu=\alpha_{s}\right)$ at NLL order include the sizable negative normalization corrections $\propto \alpha_{s}^{3} \ln ^{2} \alpha_{s}$ found in Ref. [12]. At NNLL order we find that there are no additional contributions to the anomalous dimension for $c_{1}$, so Eq. (12) remains valid. However, solving for the full NNLL $c_{1}(\nu)$ requires the NLL values of $\mathcal{V}_{c}, \mathcal{V}_{2}, \mathcal{V}_{r}$, and $\mathcal{V}_{s}$, and the NNLL value of $\mathcal{V}_{k}$. Besides $\mathcal{V}_{c}$, the coefficients at this order are not yet known. For our NNLL solution we will use $c_{1}(1)$ at $\mathcal{O}\left(\alpha_{s}^{2}\right)$ with NLL evolution in $\nu$. From previous experience in weak decays, it is not expected that the NLL (NNLL) results for $\mathcal{V}_{2}, \mathcal{V}_{r}$, and $\mathcal{V}_{s}\left(\mathcal{V}_{k}\right)$ will deviate significantly from the LL (NLL) ones. Thus, our result for the top-antitop cross 
section should yield a realistic estimate of the theoretical uncertainties at NNLL order. The LL result for $c_{2}$ is generated by ultrasoft gluons renormalizing $\boldsymbol{O}_{p, 1}$ and reads

$$
c_{2}(\nu)=-\frac{1}{6}-\frac{32}{\beta_{0}} \ln \left(\frac{\alpha_{s}\left(m \nu^{2}\right)}{\alpha_{s}(m)}\right) .
$$

The first logarithm in this series agrees with the logarithm in the matching calculation in Ref. [13].

In full QCD the expression for the normalized photon-induced cross section for heavy top-antitop production $R=\sigma_{t \bar{t}} / \sigma_{\mu^{+} \mu^{-}}$at the c.m. energy $\sqrt{s}$ is

$$
R=\frac{16 \pi}{9 s} \operatorname{Im}\left[-i \int d^{4} x e^{i q \cdot x}\left\langle 0\left|T j^{\mu}(x) j_{\mu}(0)\right| 0\right\rangle\right],
$$

where $q=(\sqrt{s}, 0)$ and $j^{\mu}$ is the vector current that produces a top-antitop pair. In vNRQCD at NNLL order the vector current correlator is replaced by the correlators of the nonrelativistic currents $\boldsymbol{O}_{p, 1}$ and $\boldsymbol{O}_{p, 2}$ evaluated for $\nu \sim \alpha_{s}$. The correlator of two $\boldsymbol{O}_{p, 1}$ currents is proportional to the coordinate space Green function $G(0,0)$ obtained from the NNLL Schrödinger in Eq. (2), and the correlator of $\boldsymbol{O}_{\boldsymbol{p}, 1}$ and $\boldsymbol{O}_{\boldsymbol{p}, 2}$ is proportional to $(E / m) G(0,0)$. To determine $G(0,0)$ at NNLL order we use a combination of numerical and analytic calculations [14]. The ultraviolet-finite contributions from the Coulomb potential (4) [denoted by $G^{c}$ in Eq. (15) below] are determined exactly using numerical techniques developed in Ref. [15]. The ultraviolet-divergent contributions from $\tilde{V}_{\delta}$, $\tilde{V}_{r}, \tilde{V}_{k}$, and the kinetic energy correction (denoted by $\delta G^{\delta}$, $\delta G^{r}, \delta G^{k}$, and $\delta G^{\mathrm{kin}}$, respectively) are computed in perturbation theory with dimensional regularization in the $\overline{\mathrm{MS}}$ scheme. This is required for consistency with the scheme used to compute the matching and running of the Wilson coefficients. By power counting, only a single insertion of these potentials is included. In deriving these results we have included the counter terms generated by renormalizing the $\boldsymbol{O}_{\boldsymbol{p}, 1}$ current [2]. These counter term graphs are sufficient to cancel all subdivergences. The remaining overall divergences are of the form $1 / \epsilon$ and $v^{2} / \epsilon$ and are canceled by renormalizing the time-ordered product of currents. The final result for the NNLL cross section in vNRQCD is

$$
\begin{aligned}
& R^{\mathrm{NNLL}}=\frac{8 \pi}{m^{2}} \operatorname{Im}\left\{c _ { 1 } ^ { 2 } \left[\left(1-v^{2}\right) G^{c}+\left(\mathcal{V}_{2}+2 \mathcal{V}_{s}\right) \delta G^{\delta}\right.\right. \\
& \left.+\mathcal{V}_{r} \delta G^{r}+\mathcal{V}_{k} \delta G^{k}+\delta G^{\mathrm{kin}}\right] \\
& \left.+2 c_{1} c_{2} v^{2} G^{c}\right\}
\end{aligned}
$$

where $v$ is the top-quark velocity and the dependence of the Wilson coefficients on $\nu$ is suppressed. The top-quark width is implemented by shifting the energy into the positive complex energy plane by $i \Gamma_{t}[16]$, which leads to the following expression for the top-quark velocity

$$
v=\left(\frac{\sqrt{s}-2 m_{t}+i \Gamma_{t}}{m_{t}}\right)^{1 / 2}
$$

We emphasize that Eq. (16) does not provide a consistent treatment of the top-quark width beyond next-to-leading order. This can be seen from the presence of $\nu$ dependent terms proportional to $\alpha_{s} \Gamma_{t} / m_{t} \ln (-i v / \nu)$ in Eq. (15) which are parametrically of NNLL order [14]. Conceptually, they indicate that further renormalization procedures are required in a consistent treatment of electroweak effects.

In the upper panel of Fig. 1 the normalized top-quark cross section is displayed in the $1 \mathrm{~S}$ mass scheme $[4,17]$ versus the c.m. energy $\sqrt{s}$, for $m_{t}^{1 \mathrm{~S}}=175 \mathrm{GeV}$, $\alpha_{s}^{\left(n_{f}=5\right)}\left(M_{Z}\right)=0.118$, and $\Gamma_{t}=1.43 \mathrm{GeV}$. The LL (dotted lines), NLL (dashed lines), and NNLL (solid lines) results are shown for $\nu=0.15,0.2$, and 0.4. From a physical point of view the appropriate choice of the subtraction parameter $\nu$ is around $\alpha_{s} \approx 0.15-0.2$. The LL curves for $\nu=0.15,0.2$, and 0.4 correspond to the upper, middle, and lower lines, respectively. The NLL and NNLL curves differ so little on the vertical scale of the figure that we refrain from labeling them. At NNLL order, the relative variation of the cross section at the peak position is $2 \%$ for $0.15<\nu<0.4$,
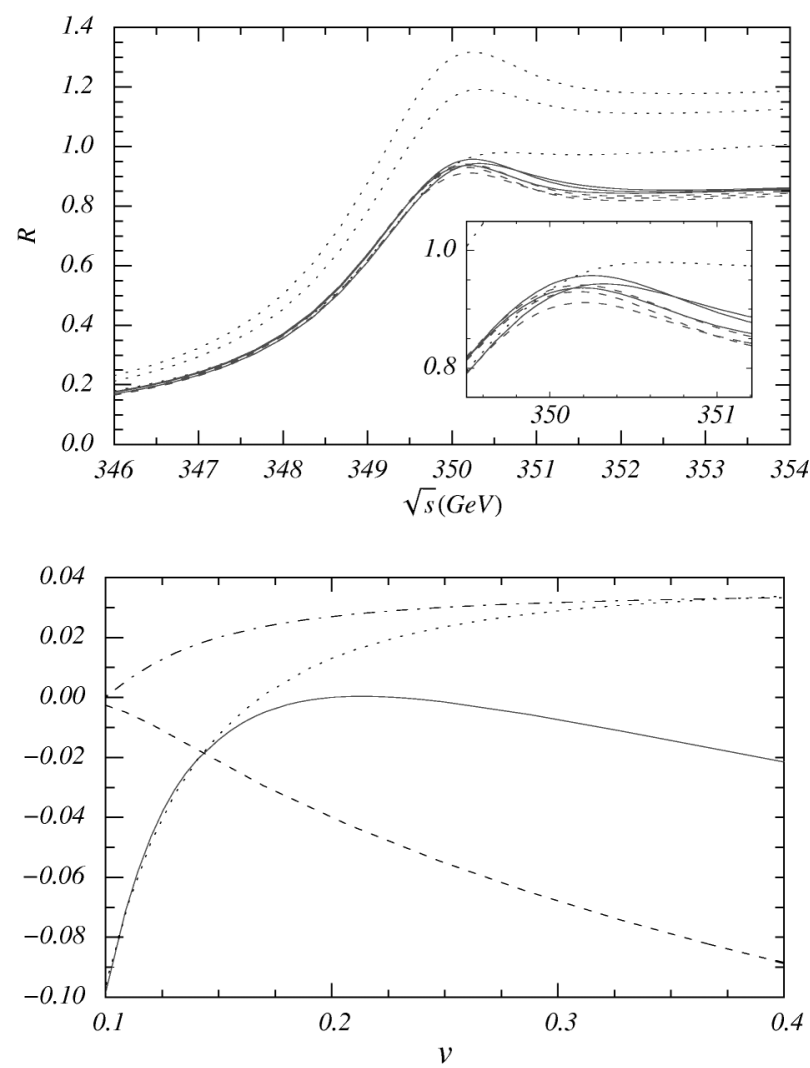

FIG. 1. The upper panel shows the normalized photon-induced cross section for $t \bar{t}$ at LL order (dotted line), NLL order (dashed line), and NNLL order (solid line) in the 1S mass scheme with $\nu=0.15,0.2$, and 0.4. The lower panel shows the $\nu$ dependence of the contributions in Eq. (15) to $R^{\mathrm{NNLL}}(\sqrt{s}=$ $350 \mathrm{GeV}$ ). The $\delta G^{k}$ contribution is shown by the dotted line, the sum of $\delta G^{\delta, r \text {, kin }}$ terms by the dot-dashed line, the terms involving $\delta G^{c}$ by the dashed line, and the sum by the solid line. 
whereas for $\sqrt{s}=(346,350,351,354) \mathrm{GeV}$ the variation is $(4 \%, 2 \%, 3 \%, 0.7 \%)$. At NLL and LL order the corresponding variations are $3 \%(2 \%, 3 \%, 2 \%, 2 \%)$ and $34 \%(28 \%, 39 \%, 27 \%, 18 \%)$, respectively. Overall, the variation of the normalization of the cross section for reasonable choices of $\nu$ and the shift due to the NNLL order corrections are an order of magnitude smaller than in previous NNLO calculations where threshold masses were employed (see Ref. [1]). The improved stability of the NNLL cross section is a consequence of the evolution of the Wilson coefficients for the potentials and the currents.

In the lower panel of Fig. 1 the contributions to the cross section at $\sqrt{s}=350 \mathrm{Gev}$ coming from $G^{c}$ (dashed line), $\delta G^{k}$ (dotted line), and the sum of $\delta G^{\delta, r, \text { kin }}$ (dot-dashed line) are displayed as a function of $\nu$. The sum of all contributions is represented by the solid line. For convenience of presentation we have subtracted the value of $R^{\mathrm{NNLL}}$ at $\nu=0.2$ from the dashed and solid curves. Whereas the individual contributions vary quite rapidly, most notably the Coulomb term $G^{c}$ and the $1 /|\mathbf{k}|$ potential term $\delta G^{k}$, there is a partial cancellation in the sum, which varies more slowly. The correlation of the Wilson coefficients that leads to this stability is a consequence of the vNRQCD renormalization-group equations that account for the correlated soft and ultrasoft running and the mixing of Wilson coefficients for $\nu<1$. We note that the corrections coming from $\tilde{V}_{k}$ quickly become negative for small $\nu$, and lead to an instability of the NNLL curve at the peak if $\nu$ is chosen smaller than the Coulombic velocity $v \simeq 0.15$. For $\nu>0.15$ multiple energy poles caused by the perturbative treatment of the potentials $\tilde{V}_{\delta}, \tilde{V}_{r}$, and $\tilde{V}_{k}$ and the kinetic energy correction do not have to be resummed because the corresponding corrections in the binding energies are an order of magnitude smaller than the top-quark width, $\Gamma_{t} \approx 1.5 \mathrm{GeV}$. However, for $\nu<0.15$ the residue of the double-pole $\mathcal{V}_{k}$ term becomes large and multiple insertions of $\tilde{V}_{k}$ at the peak have to be summed to stabilize the cross section. For $\nu<0.1$ the value of the Wilson coefficients changes rapidly due to the fact that $\mu_{U}=m_{t} \nu^{2}$ gets close to $1 \mathrm{GeV}$.

It is instructive to consider the size of some current correlator corrections from beyond NNLL for $\nu>0.15$. The corrections arising from two insertions of $\tilde{V}_{\delta}$ are smaller than $1 \%$ for $\nu>0.15$. In Ref. [18] the corrections to the square of the heavy quarkonium (nS) wave function at the origin arising from the emission and reabsorption of an ultrasoft gluon were determined, which can be taken as an estimate for ultrasoft corrections to the cross section. To be compatible with our calculations the $\overline{\mathrm{MS}}$ parameter used in Ref. [18] has to be replaced by the ultrasoft scale $\mu_{U}$. For the ground state $(n=1)$ one finds that the corrections amount to about $2 \%$ for $\nu \approx \alpha_{s}$. The small size of the two corrections just mentioned strengthens our confidence that the 2\%-3\% variation of the normalization with $\nu$ at NNLL order represents a realistic estimate of the theoretical uncertainties. Using the prescription given in Ref. [19] the relative corrections to the normalization of the cross section from a $115 \mathrm{GeV}$ standard-model Higgs boson are $5 \%-8 \%$ for energies near the threshold. This is larger than the remaining uncertainty of the NNLL cross section.

A. H. is supported in part by the EU Fourth Framework Program "Training and Mobility of Researchers," Network "Quantum Chromodynamics and Deep Structure of Elementary Particles," Contract No. FMRX-CT98-0194 (DG12-MIHT). I.S. is supported in part by NSERC of Canada, and A.M. and I.S. are supported in part by the U.S. Department of Energy under Contract No. DOE-FG03-97ER40546.

[1] A. H. Hoang et al., Eur. Phys. J. C 3, 1 (2000).

[2] M.E. Luke, A. V. Manohar, and I. Z. Rothstein, Phys. Rev. D 61, 074025 (2000).

[3] A. V. Manohar and I. W. Stewart, Phys. Rev. Lett. 85, 2248 (2000); A. V. Manohar, J. Soto, and I. W. Stewart, Phys. Lett. B 486, 400 (2000).

[4] A. H. Hoang and T. Teubner, Phys. Rev. D 60, 114027 (1999).

[5] A. H. Hoang, A. V. Manohar, and I. W. Stewart, Report No. UCSD/PTH 2000-24; see also A. Pineda and J. Soto, Phys. Lett. B 495, 323 (2000).

[6] Y. Schröder, Phys. Lett. B 447, 321 (1999); M. Peter, Phys. Rev. Lett. 78, 602 (1997).

[7] S. Titard and F. J. Yndurain, Phys. Rev. D 49, 6007 (1994).

[8] A. V. Manohar and I. W. Stewart, Phys. Rev. D 63, 054004 (2001).

[9] A. V. Manohar and I. W. Stewart, Phys. Rev. D 62, 014033 (2000).

[10] A. Czarnecki and K. Melnikov, Phys. Rev. Lett. 80, 2531 (1998); see also A.H. Hoang, Phys. Rev. D 56, 7276 (1997).

[11] M. Beneke, A. Signer, and V. A. Smirnov, Phys. Rev. Lett. 80, 2535 (1998).

[12] B. A. Kniehl and A. A. Penin, Nucl. Phys. B577, 197 (2000).

[13] M. Luke and M. J. Savage, Phys. Rev. D 57, 413 (1998).

[14] A.H. Hoang and T. Teubner, Phys. Rev. D 58, 114023 (1998).

[15] M. Jeżabek, J.H. Kühn, and T. Teubner, Z. Phys. C 56, 653 (1992).

[16] V.S. Fadin and V. A. Khoze, JETP Lett. 46, 525 (1987).

[17] A. H. Hoang, Z. Ligeti, and A. V. Manohar, Phys. Rev. Lett. 82, 277 (1999); Phys. Rev. D 59, 074017 (1999).

[18] B.A. Kniehl and A.A. Penin, Nucl. Phys. B563, 200 (1999).

[19] R. Harlander, M. Jeżabek, and J. H. Kühn, Acta Phys. Pol. 27, 1781 (1996). 